\title{
Exponential input-to-state stability of composite stochastic systems
}

\author{
Fakhreddin Abedi*, Wah June Leong and Sarkhosh Seddighi Chaharborj
}

${ }^{*}$ Correspondence:

f_abedi1352@yahoo.com

Department of Mathematics,

Faculty of Science, Universiti Putra

Malaysia, Serdang, Selangor 43400

UPM, Malaysia

\begin{abstract}
Sufficient conditions for the exponential input-to-state stability in probability in $r$ th mean and for the almost sure exponential input-to-state stability in probability of a composite stochastic system are established. Illustrating example is provided to validate our results.
\end{abstract}

MSC: 60H10; 93C10; 93D05; 93D15; 93D21; 93E15

Keywords: composite stochastic control system; control Lyapunov function; exponential input-to-state stability in probability

\section{Introduction}

The stability of deterministic control systems has been widely developed in the past few decades (see, for instance, Sontag and Wang [1], Grune [2], Karafyllis and Tsinias [3], Ning et al. [4]). In these papers, necessary and sufficient conditions for input-to-state stability at the equilibrium state of deterministic control systems were provided. On the other hand, the asymptotic stability, exponential stability and input-to-state stability in probability at the equilibrium state of stochastic control systems (SCS) were established by Mao [5], Liu and Raffool [6], Lan and Dang [7], Abedi et al. [8, 9], Abedi and Leong [10], Khasminiskii [11], Kushner [12], Tsinias [13], Ito [14], Liu et al. [15], Duan et al. [16] and Yu and Xie [17]. Particularly, Ito [14] developed the properties of input-to-state stability and gave the design of controller by using nonlinear small-gain conditions. While Liu et al. [15] discussed the property of practical stochastic input-to-state stability and its application to stability of cascaded nonlinear systems. The concept of integral input-to-state stability to the stochastic nonlinear systems was generalized by Yu and Xie [17] and Duan et al. [16] and state feedback controller that guarantees that all the signals of the resulting closed-loop system are bounded almost surely was also given.

Michel [18] developed exponential stability in probability of a continuous and discrete parameters composite stochastic system. Later, Boulanger [19] derived sufficient conditions for asymptotic stability in probability and exponential stability in mean square for a special case of our composite stochastic control system (CSCS). The sufficient conditions for exponential stability in probability of nonlinear stochastic systems, which are the special case of our CSCS, were established by Rusinek [20].

The purpose of the paper is to fill the gaps in the literature by studying the exponential input-to-state stability in probability in $r$ th mean (REISSP) and the almost sure exponential input-to-state stability in probability (AEISSP) of a CSCS. We take this fact into account to extend the REISSP and AEISSP results established by Spiliotis and Tsinias [21]

(c) 2013 Abedi et al.; licensee Springer. This is an Open Access article distributed under the terms of the Creative Commons Attribution License (http://creativecommons.org/licenses/by/2.0), which permits unrestricted use, distribution, and reproduction in any medium, provided the original work is properly cited. 
for SCS to the broader class of CSCS driven by two independent Wiener processes that were considered in Boulanger [19] and Rusinek [20]. The main results of our work enable us to derive sufficient conditions for REISSP and AEISSP of the CSCS. We also establish the existence of an explicit formula for a feedback law exhibiting the REISSP and AEISSP property.

The rest of the paper is organized as follows. In Section 2, we introduce a wider class of CSCS and we also recall some basic definitions and results relating the REISSP and AEISSP property. Finally in Section 3, we use the results of Section 2 and prove that the CSCS satisfies the REISSP and AEISSP property. We also provide a numerical example to illustrate our results.

\section{Assumptions and preliminaries}

The aim of this section is to introduce a class of stochastic differential systems (SDS) and recall some basic definitions and theorems concerning an exponential control Lyapunov function for the exponential stability in probability of this system that we are dealing with in the rest of the paper. We also focus on the properties of the exponential control Lyapunov function, which plays an important role in the exponential stability, in Section 3.

Let $(\Omega, F, P)$ be a complete probability space, and denote by $\left(w_{t}\right)_{t \geq 0}$ a standard $\mathbb{R}^{m}$-valued Wiener process defined on this space.

Consider the multi-input SDS in $\mathbb{R}^{n}$

$$
d x=f(x) d t+h(x) d w_{t}
$$

where the state space is $\mathbb{R}^{n}, f$ and $h$ are Lipschitz functionals mapping from $\mathbb{R}^{n}$ into $\mathbb{R}^{n}$ and $\mathbb{R}^{n \times m}$, respectively, vanishing at the origin and there exists a nonnegative constant $C$ such that for any $x \in \mathbb{R}^{n}$, the following growth condition holds:

$$
|f(x)-f(y)|+|h(x)-h(y)| \leq C|x-y|, \quad \forall x, y \in \mathbb{R}^{n} .
$$

Assume that for a $C^{2}$ function $\Phi: \mathbb{R}^{n} \rightarrow \mathbb{R}^{+}$,

$$
\mathbf{D} \Phi(x)=\sum_{i=1}^{n} f^{i}(x) \frac{\partial \Phi(x)}{\partial x_{i}}+\frac{1}{2} \sum_{i, j=1}^{n} \sum_{k=1}^{m} h_{k}^{i}(x) h_{k}^{j}(x) \frac{\partial^{2} \Phi(x)}{\partial x_{i} \partial x_{j}}
$$

is the infinitesimal generator for the stochastic process solution of SDS (1). Then we can recall the definition of exponential stability in probability in mean square and the stochastic version of the converse Lyapunov theorem established by Khasminskii [11] as follows.

Definition 2.1 We say that the origin is exponentially stable in probability in mean square with respect to SDS (1) if and only if there exist constants $c_{1}, c_{2}>0$ such that

$$
E\left(\left|x_{t}\left(t, t_{0}, x\right)\right|^{2}\right) \leq c_{1}|x|^{2} e^{-c_{2}\left(t-t_{0}\right)}, \quad \forall x \in \mathbb{R}^{n}, t \geq t_{0} .
$$

Theorem 2.2 Suppose that the origin is exponentially stable in probability in mean square with respect to SDS (1). Then there exist a Lyapunov function $\Phi: \mathbb{R}^{n} \rightarrow \mathbb{R}^{+}$and positive constants $a_{i}, 1 \leq i \leq 5$, such that

$$
a_{1}|x|^{2} \leq \Phi(x) \leq a_{2}|x|^{2}
$$




$$
\begin{aligned}
& |\nabla \Phi(x)| \leq a_{3}|x|, \quad\left|\nabla^{2} \Phi(x)\right| \leq a_{4}, \\
& \mathbf{D} \Phi(x)=\sum_{i=1}^{n} f^{i}(x) \frac{\partial \Phi(x)}{\partial x_{i}}+\frac{1}{2} \sum_{i, j=1}^{n} \sum_{k=1}^{m} h_{k}^{i}(x) h_{k}^{j}(x) \frac{\partial^{2} \Phi(x)}{\partial x_{i} \partial x_{j}} \leq-a_{5}|x|^{2},
\end{aligned}
$$

where $\nabla$ is the gradient operator and $\mathbf{D}$ is given in (3).

Assume that $\left(\beta_{t}\right)_{t \geq 0}$ is a standard $\mathbb{R}^{q}$-valued Wiener process defined on a complete probability space $(\Omega, F, P)$. Now, consider the multi-input SCS in $\mathbb{R}^{r}$

$$
d v=F(v, u) d t+G(v, u) d \beta_{t}
$$

where the state space is $\mathbb{R}^{r}$ and the following conditions hold:

(1) $u$ is an $\mathbb{R}^{p}$-valued measurable control law,

(2) $F$ and $G$ are Lipschitz functionals mapping from $\mathbb{R}^{r} \times \mathbb{R}^{p}$ into $\mathbb{R}^{r}$, and $\mathbb{R}^{r} \times \mathbb{R}^{q}$, respectively, such that they are vanishing at the origin and there exists constant $C \geq 0$ such that for any $v \in \mathbb{R}^{r}$ and $u \in \mathbb{R}^{p}$, the following growth condition holds:

$$
|F(v, u)|+|G(v, u)| \leq C(1+|v|+|u|) .
$$

Under restriction on growth (8) (see, for instance, Arnold [22]), for every input $u \in \mathbb{R}^{p}$, $v_{0} \in \mathbb{R}^{r}$ and $t_{0} \in \mathbb{R}$, there exists a unique solution $V\left(t, t_{0}, v_{0}, u\right)$ of (7) starting from $v_{0}$ at time $t=t_{0}$ which is defined for all $t \geq t_{0}$ and almost all $\beta \in \Omega$. In the following we assume $V(t)=V\left(t, t_{0}, v, u\right)$ and we recall the definition of RESP, AESP and the stochastic version of the converse Lyapunov theorem proved by Spiliotis and Tsinias [21] as follows.

Definition 2.3 We say that the origin is RESP with respect to SCS (7) for some $r>0$ if and only if there exist constants $c_{1}, c_{2}>0$ such that

$$
E\left(|V(t)|^{r}\right) \leq c_{1}|v|^{r} e^{-c_{2}\left(t-t_{0}\right)}, \quad \forall v \in \mathbb{R}^{r}, t \geq t_{0}
$$

Definition 2.4 SCS (7) is said to satisfy the exponential Lyapunov condition if there exists a Lyapunov function $\Phi: \mathbb{R}^{r} \rightarrow \mathbb{R}^{+}$of class $C^{2}\left(\mathbb{R}^{r} \backslash\{0\}\right)$ and positive constants $a_{i}, 1 \leq i \leq 5$, such that

$$
\begin{aligned}
& a_{1}|v|^{r} \leq \Phi(v) \leq a_{2}|v|^{r}, \\
& |\nabla \Phi(v)| \leq a_{3}|v|^{r-1}, \quad\left|\nabla^{2} \Phi(v)\right| \leq a_{4}|v|^{r-2}, \\
& \mathbf{Y} \Phi(v)=\sum_{i=1}^{n} F(v, u) \frac{\partial \Phi(v)}{\partial v_{i}}+\frac{1}{2} \sum_{i, j=1}^{n} G(v, u) G(v, u)^{T} \frac{\partial^{2} \Phi(v)}{\partial v_{i} \partial v_{j}} \leq-a_{5}|v|^{r},
\end{aligned}
$$

where $\mathbf{Y}$ is the infinitesimal generator for the stochastic process solution of SCS (7).

Theorem 2.5 Suppose that the origin is RESP with respect to SCS (7). Then there exists a Lyapunov function $\Phi: \mathbb{R}^{r} \rightarrow \mathbb{R}^{+}$of class $C^{2}\left(\mathbb{R}^{r} \backslash\{0\}\right)$ which satisfies all conditions (10)-(12). 
Definition 2.6 We say that the origin is AESP with respect to SCS (7) if and only if there exist a constant $c>0$ and a random variable $0 \leq B_{v, u}<\infty$ such that

$$
|V(t)| \leq B_{v, u} e^{-c\left(t-t_{0}\right)}, \quad \forall v \in \mathbb{R}^{r}, t \geq t_{0} .
$$

Note that Definition 2.3 and Theorem 2.5 in Spiliotis and Tsinias [21] are an extension of Definition 2.1 and Theorem 2.2, respectively, established in Khasminskii [11]. Now, we recall the definition of REISSP and AEISSP exposed in [21] as follows.

Definition 2.7 We say that the origin is REISSP with respect to SCS (7) if there exist a positive definite function $\gamma: \mathbb{R}^{+} \rightarrow \mathbb{R}^{+}$and constants $c_{1}, c_{2}>0$ such that (9) holds and

$$
|u(t)| \leq \gamma(|V(t)|), \quad \forall v \in \mathbb{R}^{r}, t \geq t_{0} .
$$

Definition 2.8 We say that the origin is AEISSP with respect to SCS (7) if, there exist a positive definite function $\gamma: \mathbb{R}^{+} \rightarrow \mathbb{R}^{+}$, constant $c>0$, and a random variable $B_{v, u}<\infty$ such that (13) and (14) hold for any $v \in \mathbb{R}^{r}$, and $t \geq t_{0}$.

We shall now turn the attention to a broader class of CSCS and provide some results related to the REISSP and AEISSP of this system.

Let us first introduce a general composite stochastic system.

Assume that $\left\{\beta_{t}, t \in \mathbb{R}^{+}\right\}$is a standard $\mathbb{R}^{q}$-valued Wiener process defined on a complete probability space $(\Omega, F, P)$ independent of a standard $\mathbb{R}^{m}$-valued Wiener process $\left\{w_{t}, t \in\right.$ $\mathbb{R}^{+}$. Consider the multi-input CSCS

$$
\left\{\begin{array}{l}
d x=(f(x)+g(x, v) D v) d t+(h(x)+q(x, v) D v) d w_{t}, \\
d v=F(v, u) d t+G(v, u) d \beta_{t}
\end{array}\right.
$$

where the following conditions hold:

(H1) $x \in \mathbb{R}^{n}, v \in \mathbb{R}^{r}$, and $D$ is a matrix function with value in $M_{r \times r}(\mathbb{R})$,

(H2) $f$ and $h$ are functionals in $C^{2}\left(\mathbb{R}^{n}, \mathbb{R}^{n}\right)$ and $C^{2}\left(\mathbb{R}^{n}, \mathbb{R}^{n \times m}\right)$, respectively, such that $f(0)=h(0)=0$,

(H3) $g: \mathbb{R}^{n} \times \mathbb{R}^{r} \rightarrow \mathbb{R}^{n \times r}$ and $q: \mathbb{R}^{n} \times \mathbb{R}^{r} \rightarrow \mathbb{R}^{n \times m}$ are Lipschitz functionals such that there exists a nondecreasing scalar function $\alpha(|v|) \geq 0$ bounded for all $v$ such that

$$
|g(x, v)|+|q(x, v)| \leq \alpha(|v|)|x|, \quad \forall(x, v) \in \mathbb{R}^{n} \times \mathbb{R}^{r},
$$

(H4) $u$ is an $\mathbb{R}^{p}$-valued measurable control law,

(H5) $F: \mathbb{R}^{r} \times \mathbb{R}^{p} \rightarrow \mathbb{R}^{r}$ and $G: \mathbb{R}^{r} \times \mathbb{R}^{p} \rightarrow \mathbb{R}^{n \times q}$ are Lipschitz functionals, vanishing at the origin and there exists a nonnegative constant $C$ such that the following growth condition holds:

$$
|F(v, u)|+|G(v, u)| \leq C(1+|v|+|u|), \quad \forall(v, u) \in \mathbb{R}^{r} \times \mathbb{R}^{p} .
$$

Suppose that there exist functionals $F_{1}: \mathbb{R}^{r} \rightarrow \mathbb{R}^{r}, F_{2}: \mathbb{R}^{r} \rightarrow \mathbb{R}^{r \times p}, G_{1}: \mathbb{R}^{r} \rightarrow \mathbb{R}^{r \times q}$ and $G_{2}: \mathbb{R}^{r} \rightarrow \mathbb{R}^{r \times p \times q}$ such that

$$
F(v, u)=F_{1}(v)+F_{2}(v) u, \quad G(v, u)=G_{1}(v)+G_{2}(v) u,
$$


and

$$
d v=\left(F_{1}(v)+F_{2}(v) u\right) d t+\left(G_{1}(v)+G_{2}(v) u\right) d \beta_{t}
$$

for any $(v, u) \in \mathbb{R}^{r} \times \mathbb{R}^{p}$. Then CSCS (15) is rewritten as

$$
\left\{\begin{array}{l}
d x=(f(x)+g(x, v) D v) d t+(h(x)+q(x, v) D v) d w_{t}, \\
d v=\left(F_{1}(v)+F_{2}(v) u\right) d t+\left(G_{1}(v)+G_{2}(v) u\right) d \beta_{t} .
\end{array}\right.
$$

By considering the results on EISSP exposed in [21], we can establish the following outcome.

Lemma 2.9 CSCS (17) satisfies the REISSP and AEISSP property if and only if $0 \in \mathbb{R}^{n}$ is RESP and AESP, respectively, for the system

$$
\left\{\begin{array}{l}
d x=(f(x)+g(x, v) D v) d t+(h(x)+q(x, v) D v) d w_{t}, \\
d v=\left(F_{1}(v)+F_{2}(v) \gamma(|v|) \theta\right) d t+\left(G_{1}(v)+G_{2}(v) \gamma(|v|) \theta\right) d \beta_{t},
\end{array}\right.
$$

where $\theta \in I=\left\{\theta \in \mathbb{R}^{p}:|\theta| \leq 1\right\}$.

Proof The proof of this lemma is a direct consequence of Definitions 2.3, 2.6-2.8 and the fact that each solution $(x(t), v(t))$ of (18) that corresponds to some $u(\cdot)$ coincides with the solution of (17) with the same initial $x_{0}, v_{0}$ and $t_{0}$ and corresponding to $u=\gamma(|v|) \theta$, namely, satisfying (14). Conversely, each solution $(x(t), v(t))$ of (17) under restriction (14) is a solution of (18) with input $\theta=u / \gamma(|v|)$ and the same initial value.

In the next section we establish the state feedback law that guarantees the satisfaction of the REISSP and AEISSP property for CSCS (17).

\section{Main stability results}

The REISSP and AEISSP results proved in this paper employ the technique which is a combination of those by Sontag and Wang [1,23], i.e., decomposition for a deterministic control system, and Spiliotis and Tsinias [21], i.e., decomposition in stochastic case for the SCS. We use these decompositions to obtain the existing exponential stability results for the CSCS. In the following theorem, we assume that the functions $g$ and $q$ are bounded on $\mathbb{R}^{n} \times \mathbb{R}^{p}$ and $U$ is the set of admissible control. To establish sufficient conditions for REISSP and AEISSP of CSCS (17), we use Lemma 2.9 and show that the origin is RESP and AESP for CSCS (18). Theorem 3.1 can be viewed as a stochastic extension of Proposition 4.1 and Theorem 4.1 stated in Spiliotis and Tsinias [21] and Boulanger [19], respectively, to a general composite stochastic system. Both the results and the tools used in this theorem, however, are different from those in $[19,21]$. Furthermore, we can consider the exponential stability results of Boulanger [19] as a special case of our REISSP results (Theorem 3.1) where $r=2$.

Theorem 3.1 Consider CSCS (17). Assume that the function $\gamma: \mathbb{R}^{+} \rightarrow \mathbb{R}^{+}$is positive definite with bounded first derivative $\gamma^{(1)}$, and for all $|u| \leq \gamma(|v|)$, there exists a $C^{2}\left(\mathbb{R}^{r} \backslash\{0\}\right)$ function $\Phi$ satisfying the exponential Lyapunov condition. Furthermore, assume that

$$
D v=F_{2}(v)^{T} \nabla \Phi_{2}(v)+\nabla \Phi_{2}(v) G_{2}(v)^{T}
$$


Then the state control law

$$
k(x, v)=k_{1}(v)-g(x, v)^{T} \nabla \Phi_{1}(x)-q(x, v)^{T} \nabla \Phi_{1}(x),
$$

where $\Phi_{1}$ is a smooth Lyapunov function corresponding to SDS (1) and $k_{1}: \mathbb{R}^{r} \rightarrow \mathbb{R}^{p}$ is a state control law corresponding to the resulting closed-loop system deduced from (16), renders CSCS (17) satisfying the REISSP and AEISSP property.

Proof For the proof of this theorem, we shall use Lemma 2.9 and show that REISSP and AISSP are satisfied for CSCS (17) if and only if the origin is RESP and AESP for CSCS (18). Assume that the function $\Phi(V(t))$ satisfies the exponential Lyapunov condition with respect to CSCS (17). Obviously, by our hypothesis, the function $\Phi(V(t))$ satisfies the exponential Lyapunov condition with respect to CSCS (18). We now show that the origin satisfies RESP and AESP for CSCS (18). First we prove that the equilibrium of CSCS (18) is satisfied in the RESP property. Since the exponential Lyapunov condition is fulfilled for CSCS (18), there exists a Lyapunov function $\Phi(V(t))$ and positive constants $a_{i}, 1 \leq i \leq 5$, such that (10)-(12) hold. Consider the composite function

$$
\Phi(V(t))=\Phi_{1}(x)+\Phi_{2}(v), \quad \forall(x, v) \in \mathbb{R}^{n} \times \mathbb{R}^{r}
$$

where $\Phi_{1}$ and $\Phi_{2}$ are the Lyapunov functions corresponding to SDS (1) and SCS (16), respectively. By substituting $\theta(x, v)=\frac{k(x, v)}{\gamma(|v|)}$ into the closed-loop system deduced from CSCS (18), we have

$$
\begin{aligned}
d x= & (f(x)+g(x, v) D v) d t+(h(x)+q(x, v) D v) d w_{t}, \\
d v= & \left(F_{1}(v)+F_{2}(v) k_{1}(v)-F_{2}(v) g(x, v)^{T} \nabla \Phi_{1}(x)\right. \\
& \left.-F_{2}(v) q(x, v)^{T} \nabla \Phi_{1}(x)\right) d t+\left(G_{1}(v)+G_{2}(v) k_{1}(v)\right. \\
& \left.-G_{2}(v) g(x, v)^{T} \nabla \Phi_{1}(x)-G_{2}(v) q(x, v)^{T} \nabla \Phi_{1}(x)\right) d \beta_{t} .
\end{aligned}
$$

Denoting $\mathbf{D}_{v}$ as the infinitesimal generator of the stochastic process solution of the resulting closed-loop system (22), we obtain

$$
\begin{aligned}
\mathbf{D}_{v} \Phi & (V(t)) \\
= & \mathbf{D} \Phi_{1}(x)+\nabla \Phi_{1}(x)^{T} g(x, v) D v+\nabla \Phi_{1}(x)^{T} q(x, v) D v+\mathbf{Y}_{2} \Phi_{2}(v) \\
& -\nabla \Phi_{1}(v)^{T} F_{2}(v) g(x, v)^{T} \nabla \Phi_{1}(x) \\
& -\nabla \Phi_{2}(v)^{T} F_{2}(v) q(x, v)^{T} \nabla \Phi_{1}(x) \\
& -\nabla \Phi_{2}(v)^{T} G_{2}(v) g(x, v)^{T} \nabla \Phi_{1}(x) \\
& -\nabla \Phi_{2}(v)^{T} G_{2}(v) q(x, v)^{T} \nabla \Phi_{1}(x)
\end{aligned}
$$

for any $(x, v) \in \mathbb{R}^{n} \times \mathbb{R}^{r}$. Substituting (19) into (23), we get

$$
\mathbf{D}_{v} \Phi(V(t))=\mathbf{D} \Phi_{1}(x)+\mathbf{Y}_{2} \Phi_{2}(v)
$$


Then, from (12) and (24), we get

$$
\begin{aligned}
\frac{d}{d t} E \Phi(V(t)) & =E\left(\mathbf{D}_{v} \Phi(V(t))\right)=E\left(\mathbf{D} \Phi_{1}(x)+\mathbf{Y}_{2} \Phi_{2}(v)\right) \\
& \leq-a_{5} E\left(|x|^{r}\right)-a_{5}^{\prime} E\left(|v|^{r}\right)
\end{aligned}
$$

Hence, the desired condition (9) is a direct consequence of inequalities (10) and (25). Therefore, CSCS (18) satisfies the RESP property at the origin. It turns out by Lemma 2.9 that CSCS (17) satisfies the REISSP property.

Finally, suppose that there exists a positive composite function

$$
\Phi(V(t))=\Phi_{1}(x) \exp ^{\left(\frac{a_{5}}{a_{2}} t\right)}+\Phi_{2}(v) \exp ^{\left(\frac{a_{5}^{\prime}}{a_{2}^{\prime}} t\right)}
$$

such that CSCS (18) satisfies the exponential Lyapunov condition. Then, by taking into account (10)-(12), (23), (19) and (26), we obtain

$$
\mathbf{D}_{v} \Phi(V(t))=\mathbf{D} \Phi_{1}(x) \exp ^{\left(\frac{a_{5}}{a_{2}} t\right)}+\mathbf{Y}_{2} \Phi_{2}(v) \exp ^{\left(\frac{a_{5}^{\prime}}{a_{2}^{\prime}} t\right)} \leq 0
$$

From (26)-(27) and the fact $\Phi(V(t)) \geq 0$, we have $\Phi_{t}=\Phi(V(t))$ is a supermartingale. Therefore, for some random $B_{x, v}$, it yields

$$
\lim _{t \rightarrow \infty} \Phi(V(t)) \leq B_{x, v}<\infty \quad \text { a.s. }
$$

From (28) we have $\Phi(V(t)) \leq K_{x, v}<\infty$ for all $t \geq 0$, almost surely, for some random $K_{x, v} \geq B_{x, v}$. The latter in conjunction with (10), (25) and (26) implies (13). Thus, CSCS (18) satisfies the AESP property at the origin. It turns out by Lemma 2.9 that CSCS (17) will also satisfy the AEISSP property, which completes the proof.

The following proposition (Proposition 3.2) is a stochastic extension of Proposition 4.2 stated in Spiliotis and Tsinias [21] to a general composite stochastic system.

Proposition 3.2 Consider CSCS (17). Assume that conditions (H1)-(H5) hold and the origin is RESP with zero input (namely, (9) holds with $u=0$ ). Then REISSP holds for certain linear positive definite function $\bar{\gamma}$, where, for some linear function $\bar{\gamma}$,

$$
(R E I S S P)_{\gamma} \Rightarrow(R E I S S P)_{\bar{\gamma}} \Rightarrow(A E I S S P)_{\bar{\gamma}}
$$

Proof Suppose that the origin of CSCS (17) satisfies the RESP property. From (24) it turns out, by the converse Lyapunov theorem (Theorem 2.5), that there exist positive constants $a_{i}, 1 \leq a_{i} \leq 5$, and $a_{5}^{\prime}$ such that conditions (10)-(11) hold and

$$
\mathbf{D}_{v} \Phi(V(t))=\mathbf{D} \Phi_{1}(x)+\mathbf{Y}_{2} \Phi_{2}(v) \leq-a_{5}|x|^{r}-a_{5}^{\prime}|v|^{r}, \quad v \neq 0, x \neq 0
$$

where $\mathbf{D}, \mathbf{Y}_{2}, \mathbf{D}_{v}$ are the infinitesimal generators for the stochastic process solutions of systems (1), (16), (22), respectively, and $\Phi_{1}(x), \Phi_{2}(v), \Phi(V(t))$ are the Lyapunov functions corresponding to the systems (1), (16), (22), respectively. It then follows from (29) that

$$
\mathbf{D}_{v} \Phi(V(t)) \leq-c_{5}|x|^{r}-c_{5}^{\prime}|v|^{r}, \quad \forall|u| \leq \bar{\gamma}(|x|+|v|)=k(|x|+|v|)
$$


for some positive constant $k$ and certain $0<c_{5}<a_{5}, 0<c_{5}^{\prime}<a_{5}^{\prime}$. The rest of the proof is a consequence of Theorem 3.1 and is, therefore, omitted.

Remark 3.3 Comparing our main results to the existing ones in [19-21], we can make the following summaries:

(i) Theorem 3.1 is the stochastic extension of Proposition 4.1 and Theorem 4.1 stated in Spiliotis and Tsinias [21] and Boulanger [19], respectively, to a general composite stochastic system. Both the results and the tools used in this theorem, however, are different from those in [19, 21]. Furthermore, we can consider the exponential stability results of Boulanger [19] as a special case of our REISSP results (Theorem 3.1) where $r=2$.

(ii) Proposition 3.2 is the stochastic extension of Proposition 4.2 stated in Spiliotis and Tsinias [21] to a general composite stochastic system. In addition, the existing exponential stability results established in $[19,20]$ and $[21]$ do not permit us to make a conclusion about REISSP and AEISSP for the broader class of CSCS (15) at the origin, whereas the results of this paper are still valid.

Finally, we conclude the paper by designing a numerical example to validate our results.

Example 3.4 Consider the CSCS

$$
\left\{\begin{array}{l}
d x=(-2 x+2 v) d t+(x+2 v) d w_{t}, \\
d v=(-4 v+v u) d t+(2 v+u) d \beta_{t},
\end{array}\right.
$$

where $\left\{w_{t}, t \in \mathbb{R}^{+}\right\}$and $\left\{\beta_{t}, t \in \mathbb{R}^{+}\right\}$are two independent standard real-valued Wiener processes, $x, v \in \mathbb{R}, u$ is a real-valued measurable control law, $g(x, v)=q(x, v)=2$ and $D=I$ is the identity matrix in $M_{r \times r}(\mathbb{R})$. Consider the Lyapunov function candidate

$$
\Phi(V(t))=\Phi_{1}(x)+\Phi_{2}(v)=\frac{1}{2}\left(x^{2}+v^{2}\right) .
$$

A simple calculation shows that $\Phi(V(t))$ satisfies the exponential Lyapunov condition and

$$
k_{1}(v)=-\frac{\mathbf{D}_{z} \Phi_{2}(v)}{1+\left(\sup _{1 \leq z, r \leq p} \mathbf{D}_{z, r} \Phi_{2}(v)\right)^{2}}=-\frac{4}{5}\left(v^{2}+2 v\right)
$$

is the state control law corresponding to the resulting closed-loop system deduced from

$$
d v=(-4 v+v u) d t+(2 v+u) d \beta_{t}
$$

where

$$
\begin{aligned}
& \mathbf{D}_{z} \Phi_{2}(v)=\sum_{i=1}^{n} K_{z}^{i}(v) \frac{\partial \Phi_{2}(v)}{\partial v_{i}}+\sum_{i, j=1}^{n} \sum_{k=1}^{m} M_{k}^{i} N_{k, z}^{j} \frac{\partial^{2} \Phi_{2}(v)}{\partial v_{i} \partial v_{j}}=v^{2}+2 v, \\
& \mathbf{D}_{z, r} \Phi_{2}(v)=\frac{1}{2} \sum_{i, j=1}^{n} \sum_{k=1}^{m} N_{k, z}^{i} N_{k, r}^{j} \frac{\partial^{2} \Phi_{2}(v)}{\partial v_{i} \partial v_{j}}=\frac{1}{2},
\end{aligned}
$$


and $H(v)=-4 v, K(v)=v, M(v)=2 v, N(v)=1$. (For more information about the state control law $k_{1}(v)$, one can refer to Theorem 3.2 established by Abedi et al. [8].) Then, according to Theorem 3.1, the state control law

$$
k(x, v)=k_{1}(v)-g(x, v)^{T} \nabla \Phi_{1}(x)-q(x, v)^{T} \nabla \Phi_{1}(x)=-\frac{4}{5}\left(v^{2}+2 v\right)-4 x
$$

guarantees that CSCS (30) satisfies the REISSP and AEISSP property.

\section{Conclusions}

We have developed the exponential input-to-state stability in probability of a larger class of multi-input composite system (15). We have used the stochastic version of converse Lyapunov theorems derived by Khasminiskii [11] and Spiliotis and Tsinias [21] to the concept of stochastic control Lyapunov function and extended the REISSP and the AEISSP results provided by Spiliotis and Tsinias [21] for a stochastic system to the larger class of composite stochastic systems driven by two independent Wiener processes that were considered in Boulanger [19] and Rusinek [20]. The main results of our work enable us to derive the sufficient conditions for REISSP and AEISSP of a composite stochastic system. We have also established the existence of an explicit formula of a feedback law exhibiting the REISSP and AEISSP property of a composite stochastic system.

\section{Competing interests}

The authors declare that they have no competing interests.

\section{Authors' contributions}

All authors read and approved the final manuscript.

\section{Acknowledgements}

The authors thank the referees for valuable comments and suggestions which improved the presentation of this manuscript.

Received: 13 March 2013 Accepted: 26 June 2013 Published: 11 July 2013

\section{References}

1. Sontag, DE, Wang, Y: Lyapunov characterizations of input to output stability. SIAM J. Control Optim. 39(1), 351-359 (2000)

2. Grune, L: Input-to-state dynamical stability and its Lyapunov characterization. IEEE Trans. Autom. Control 47(9), 1499-1504 (2002)

3. Karafyllis, I, Tsinias, J: A converse Lyapunov theorem for nonuniform in time global asymptotic stability and its application to feedback stabilization. SIAM J. Control Optim. 42, 936-965 (2003)

4. Ning, C, He, Y, Wu, M, Liu, Q, She, J: Input-to-state stability of nonlinear systems based on an indefinite Lyapunov function. Syst. Control Lett. 61, 1254-1259 (2012)

5. Mao, X: Exponential Stability of Stochastic Differential Equations. Dekker, New York (1994)

6. Liu, R, Raffoul, Y: Boundedness and exponential stability of highly nonlinear stochastic differential equations. Electron. J. Differ. Equ. 2009, 143 (2009)

7. Lan, TTT, Dang, NH: Exponential stability of nontrivial solutions of stochastic differential equations. Scientia, Ser. A Math. Sci. 21, 97-106 (2011)

8. Abedi, F, Abu Hassan, M, Arifin, MN: Lyapunov function for nonuniform in time global asymptotic stability in probability with application to feedback stabilization. Acta Appl. Math. 116(1), 107-117 (2011)

9. Abedi, F, Abu Hassan, M, Suleiman, M: Feedback stabilization and adaptive stabilization of stochastic nonlinear systems by the control Lyapunov function. Stoch. Int. J. Probab. Stoch. Process. 83(2), 179-201 (2011)

10. Abedi, F, Leong, JW: Dynamic robust stabilization of stochastic differential control systems. IMA J. Math. Control Inf. (2013). doi:10.1093/imamci/dns040

11. Khasminskii, ZR: Stochastic Stability of Differential Equation. Sijthoff \& Noordhoff, Alphen aan den Rijn (1980)

12. Kushner, JH: On the stability of processes defined by stochastic difference-differential equation. J. Differ. Equ. 4, 424-443 (1968)

13. Tsinias, J: Stochastic input-to-state stability and applications to global feedback stabilization. Int. J. Control 71 907-931 (1998)

14. Ito, H: State-dependent scaling problems and stability of interconnected iISS and ISS systems. IEEE Trans. Autom. Control 51(10), 1626-1643 (2006) 
15. Liu, SJ, Zhang, JF, Jiang, ZP: A notion of stochastic input-to-state stability and its application to stability of cascaded stochastic nonlinear systems. Acta Math. Appl. Sin. 24(1), 141-156 (2008)

16. Duan, $\mathrm{N}, \mathrm{Xie}, \mathrm{XJ}, \mathrm{Yu}, \mathrm{X}$ : State feedback stabilization of stochastic nonlinear systems with SilSS inverse dynamics. Int. J. Robust Nonlinear Control 21, 1903-1919 (2011)

17. $Y u, X, X i e, X J$ : Output feedback regulation of stochastic nonlinear systems with stochastic ilSS inverse dynamics. IEEE Trans. Autom. Control 55(2), 304-320 (2010)

18. Michel, NA: Stability analysis of stochastic composite systems. IEEE Trans. Autom. Control 20(2), 246-250 (1975)

19. Boulanger, C: Stabilization of a class of nonlinear composite stochastic systems. Stoch. Anal. Appl. 18(5), 723-735 (2000)

20. Rusinek, RL: String stability of singularly perturbed stochastic systems. Ann. Math. Sil. 16, $43-55$ (2003)

21. Spiliotis, J, Tsinias, J: Notions of exponential robust stochastic stability, ISS and their Lyapunov characterizations. Int. J. Robust Nonlinear Control 13,173-187 (2003)

22. Arnold, L: Stochastic Differential Equations: Theory and Applications. Wiley, New York (1974)

23. Sontag, DE, Wang, Y: On characterizations of input-to-state stability properties. Syst. Control Lett. 24, 226-231 (1995)

doi:10.1186/1687-1847-2013-208

Cite this article as: Abedi et al.: Exponential input-to-state stability of composite stochastic systems. Advances in Difference Equations 2013 2013:208.

\section{Submit your manuscript to a SpringerOpen ${ }^{\circ}$ journal and benefit from:}

- Convenient online submission

Rigorous peer review

- Immediate publication on acceptance

- Open access: articles freely available online

- High visibility within the field

- Retaining the copyright to your article 